\title{
OCCURRENCE OF CLOSTRIDIUM DIFFICILE IN INFECTIONS OF MAN
}

\author{
LOUIS DS. SMITH AND ELIZABETH O. KING
}

Department of Botany and Bacteriology, Montana State College, Bozeman, Montana, and the Communicable Disease Center, U.S. Public Health Service, Atlanta, Georgia

Received for publication January 13, 1962

\section{ABSTRACT}

Sмiтh, Louis DS. (Montana State College, Bozeman) and Elizabeth O. King. Occurrence of Clostridium difficile in infections of man. J. Bacteriol. 84:65-67. 1962-Eight strains of Clostridium difficile were isolated from cases of infection in man in seven different bacteriological laboratories. One of these was isolated from a case of gas gangrene, one from an abscess following a fractured femur, one from a blood culture from an infant, two from pleural fluid, two from peritoneal fluid, and one from an abscess in the vaginal vault. There was no evidence, in these cases, that C. difficile is pathogenic for man. All strains were typical morphologically and culturally and were lethal when inoculated intramuscularly into guinea pigs.

Clostridium difficile was first isolated by Hall and O'Toole (1935), who found it as part of the normal microbial flora of the stools of infants. Since that time there has been no report of the isolation of this organism, either from infections or as part of the normal microbial flora of the human body. It was isolated from the intestinal contents of a Weddell seal killed in the Antarctic (McBee, 1960).

During the past 4 years, seven strains isolated from infections in man by various workers in hospital laboratories have been received by the Communicable Disease Center of the U.S. Public Health Service for identification. These strains, and one isolated from a hitherto unreported case of gas gangrene in World War II, are the subject of this report.

\section{SOURCES OF THE STRAINS}

Strain 1 was isolated in November 1943 in Italy at the 15th Medical General Laboratory from a case of gas gangrene in an American soldier. The wound, which was inflicted by a shell fragment during enemy action, involved the soft tissues of the left thigh without fracture or vascular injury. Debridement was performed $40 \mathrm{hr}$ after wounding. A large amount of gas and a moderate amount of edema were noted in the muscles of the thigh 3 days later, and diagnosis of clostridial myositis was made. The patient died 5 $\mathrm{hr}$ later, $131 \mathrm{hr}$ after wounding. The specimen of muscle taken at post-mortem examination for bacteriological investigation was delayed several days in transit to the laboratory and was noted on arrival as "poor specimen." C. sporogenes and C. sphenoides were isolated from this specimen as well as $C$. difficile.

Strain 2 was isolated in August 1957 at St. Joseph's Infirmary, Atlanta, Ga., from an abscess that followed a fracture of the femur suffered in ar airplane accident by an adult male. The pre sence of gas in this abscess was noted 11 days before $C$. difficile was isolated (37 days after the fracture was sustained). An obligately anaerobic staphylococcus was also isolated from fluid aspirated from this abscess.

Strain 3 was isolated in October 1957 at the Grady Memorial Hospital, Atlanta, Ga., from a blood culture from a 5-month-old male infant who had a history of coryza, cough, and anorexia for 3 weeks. No other organism was isolated from the blood of this patient.

Strain 4 was isolated in January 1959 at the Western Pennsylvania Hospital, Pittsburgh, Pa., from pleural fluid from a 65-year-old man suffering from severe acute and chronic pleuritis. $C$. difficle was demonstrated in the pleural fluid on two occasions, 26 days apart. On neither occasion were other organisms isolated, although later in the course of the disease Staphylococcus aureus and Pseudomonas aeruginosa were found.

Strain 5 was isolated at the Mercy Hospital, Redding, Calif., in January 1959 from peritoneal fluid from a 14-year-old girl suffering from bowel obstruction, peritonitis, and a subphrenic abscess. She developed jaundice secondary to acute hemolysis and died 5 days after $C$. difficle had been isolated. No other organism was found in the peritoneal fluid. 
Strain 6 was isolated at the Georgia Baptist Hospital, Atlanta, Ga., in January 1959 from pleural fluid from a 58-year-old man with pneumothorax with pleural effusion. No other bacteria were isolated from the specimen of pleural fluid at the time of the first isolation of $C$. difficile. Later, a pseudomonad was also isolated.

Strain 7 was isolated at the Grady Memorial Hospital, Atlanta, Ga., from a fatal case of peritonitis in a 73-year-old woman, who was also suffering from acute and chronic pyelonephritis. The peritonitis apparently was secondary to thrombosis of the mesenteric arteries and an infarcted small bowel.

Strain 8 was isolated in April 1961 at St. John's Hospital, Santa Monica, Calif., from an abscess in the vaginal vault of a 45-year-old woman. $C$. difficile was accompanied by anaerobic streptococci and a nonspore-forming obligately anaerobic bacterium in this abscess.

MORPHOLOGICAL AND CULTURAL CHARACTERISTICS

These eight strains were relatively uniform in their morphological and cultural characteristics. All were long, thin, motile gram-positive rods with oval terminal spores that did not markedly swell the rods. Spores were usually apparent on the second day of cultivation but appeared at this time to be subterminal in position because of the presence of a terminal cap of protoplasm that disappeared on further incubation. The Gram stain tended to be lost when cultures were incubated for more than 1 day. Colonies on bovine blood agar were 2 to $3 \mathrm{~mm}$ in diameter, slightly raised, white, opaque, with a glossy surface and entire margin. There was no hemolysis in the agar surrounding or under the colonies. On Willis and Hobbs' (1959) egg yolk-lactose-milk agar containing $100 \mu \mathrm{g}$ per $\mathrm{ml}$ of neomycin the colonies were smaller, from 0.5 to $1.5 \mathrm{~mm}$ in diameter, raised, glossy, with irregular margins. There was no evidence on this medium of the fermentation of lactose, the digestion of casein, or of the production of lecithinase. In the usual fermentation base medium, glucose, xylose, salicin, mannitol, and sorbitol were fermented; maltose, lactose, sucrose, and glycerol were not. Gelatin was not liquefied; iron-milk was not changed; nitrate was not reduced to nitrite; and indole was not formed.

Antibiotic susceptibility tests were performed using commercially prepared paper discs on surface-inoculated blood agar medium. These cultures were incubated for 3 days before examination. In these tests, the growth of all strains was found to be inhibited by penicillin, chlortetracycline, and chloramphenicol. Growth of all but strain 8 was inhibited by novobiocin; growth of strains 6 and 7 was inhibited by erythromycin. There was no appreciable inhibition of the growth of any strain by streptomycin, neomycin, colistin, or oleandomycin.

The intramuscular inoculation of $0.5 \mathrm{ml}$ of a 2-day-old broth culture of each of these strains into guinea pigs was followed by slight to moderate swelling at the site of inoculation and the death of the animals in 2 to 8 days. Little change from normal was noted at the post-mortem examination of these animals. There was no hemorrhage or other evidence of necrotic action, and $C$. difficile could not be regularly isolated from the site of inoculation in animals which survived more than a few days. Apparently, the death of these guinea pigs was brought about by the toxin introduced with the inoculum.

\section{DISCUSSION}

It seems likely that the human body was the source of these strains. Soil contamination, either direct or indirect, may have been involved in the gas gangrene of the thigh, but it seems unlikely to have been concerned in the other infections. The intestine may have been the source of strains 5 and 7 , and possibly strains 1 and 2 also, for the possibility of wounds of the thigh becoming contaminated with bacteria of fecal origin is relatively high. This would be in agreement with the other information that we have on the habitat of this organism, the finding of Hall and O'Toole (1935) that it was present in the intestinal tract of normal infants. Nevertheless, strains $3 ; 4$, and 6 were isolated from infections in which it seems likely that the source of the organisms was the respiratory tract. The isolation of strain 8 from an abscess of the vaginal vault indicates that $C$. difficile may, occasionally at least, form part of the microbial flora of the vagina.

It is not certain that $C$. difficile was responsible for the death of any of the three cases that terminated fatally. Although it was the only pathogenic clostridium isolated from the case of gas gangrene, the delay before bacteriological examination of the specimen makes it possible that more virulent bacteria may have originally been present but died during transit. The case 
from which strain 5 was isolated also terminated fatally and no other pathogenic bacterium was noted to be present. Nevertheless, the hemolytic type of jaundice that occurred in this patient indicates that $C$. difficile cannot readily be incriminated here, for it is not known to be hemolytic under any circumstances. The case from which strain 7 was isolated also was not one from which it could be clearly concluded that $C$. difficile was responsible for death, for too many complicating factors were present.

In considering the nonfatal cases, there is little evidence to indicate that $C$. difficile was anything other than a secondary invader, growing, but doing no harm except perhaps locally. This seemed to be the situation even when the organism was present for a period long enough for toxin production to have taken place, as it was in the cases from which strains 2 and 4 were isolated. It may be tentatively concluded that either $C$. difficile does not regularly produce its characteristic toxin when it grows in the human body, or that the human body is not markedly sensitive to the lethal action of this toxin.

\section{LITERATURE CITED}

Hall, I. C., and E. O'Toole. 1935. Intestinal flora in new-born infants with a description of a new pathogenic anaerobe, Bacillus difficilis. Am. J. Diseases Children 49:390-402.

MCBEE, R. H. 1960. Intestinal flora of some antarctic birds and mammals. J. Bacteriol. 79: $311-312$.

Willis, A. T., and G. Hobrs. 1959. Some new media for the isolation and identification of clostridia. J. Pathol. Bacteriol. 77:511-521. 